\title{
The Application of the Optimal GM $(1,1)$ Model for Heating Load Forecasting
}

\author{
Fan Guojuan ${ }^{1, a}$, Li Bo ${ }^{2, b}$, Mu Wanquan ${ }^{3, \mathrm{c}}$ and Ji Cun ${ }^{4, \mathrm{~d}}$ \\ ${ }^{1}$ Department of Information Engineering, Shandong Communication \& Media College, Jinan, China \\ ${ }^{2}$ Department of Information Engineering, Shan-dong Polytechnic, Jinan, China \\ ${ }^{3}$ Organization and personnel department, Shandong Polytechnic, Jinan, China \\ ${ }^{4}$ School of computer Science \& Technology, Shandong University, Jinan, China

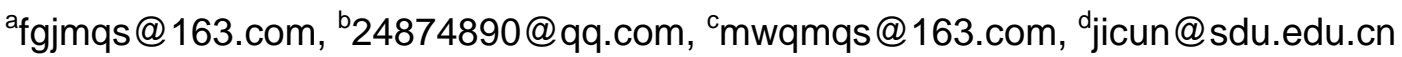

Keywords: optimal GM $(1,1)$ model; grey prediction; heating load forecasting.

\begin{abstract}
Heating load forecasting of municipal steam heating system is an important part of the smart city. According to the forecast results, heating companies can arrange production reasonably and provide heating uniform and stable to improve energy efficiency. For the features of the time series of heating monitor data, we present the optimal GM $(1,1)$ model based on the metabolic GM $(1$, 1) model. Results from theoretical analysis, simulations, and experiments show that the performance of the optimal GM $(1,1)$ model forecasting is effective with smaller error, robustness against outliers and able to meet forecast demand.
\end{abstract}

\section{Introduction}

Heating load is the most important parameter in the central heating system. Accurate forecasting of heating load can provide the basis for optimizing adjust the amount of heating supply. With the help of forecasting, the heat source enterprises can formulate reasonable heating plan to improve energy efficiency, improve the operation efficiency of heating system and reduce the cost of heating enterprises ${ }^{[1]}$. Forecasting by using large amount of heating monitoring time series data can helps the heat source enterprises arrange production reasonably and provide heating uniform and stable to improve energy efficiency. It has important research value and practical significance.

The GM(1,1) model is a classical grey forecasting model which is capable of modeling and forecasting of small sample and poor information system ${ }^{[2]}$. It has been widely used in the fields of economy, management and engineering technology ${ }^{[3-6]}$, and has achieved good results. This paper presents a new optimal GM $(1,1)$ model for heating load forecasting, reports the experiment results and analysis of adequacy of the optimal GM $(1,1)$ model.

\section{The Typical GM $(1,1)$ Model}

Original GM (1, 1) Model. The original GM $(1,1)$ model was introduced by Julong Deng, which was the most influential and the most widely used grey forecasting model in the present. It can be used to model for non-exponential growth sequence ${ }^{[2]}$. The $\mathrm{GM}(1,1)$ model constructing process is described below ${ }^{[6]}$ :

Denote the original data sequence is: $X^{(0)}=\left\{X^{(0)}(1), X^{(0)}(2), \ldots, X(\mathrm{n})\right\}$. The AGO formation of $\mathrm{X}^{(0)}$ is defined as: $X^{(1)}=\left\{X^{(1)}(1), X^{(1)}(2), \ldots, X^{(1)}(\mathrm{n})\right\}$, where $X^{(1)}(\mathrm{k})=\sum_{i=1}^{k} X^{(0)}(1), \mathrm{k}=$ $1,2,3, \ldots, \mathrm{n}$. The $\mathrm{GM}(1,1)$ model can be constructed by establishing a first order differential equation for $\mathrm{X}^{(1)}(\mathrm{k})$ as: $\mathrm{GM}(1,1): d X^{(1)}(\mathrm{k}) / d k+\alpha X^{(1)}(\mathrm{k})=\mu \quad$. Its gray differential equation (differential form) is: $X^{(0)}(k)+a z^{(1)}(k)=\mu, k=2,3, \ldots, n$. The identification of the constant a and $\mu$ in the equation constitute a vector $\hat{\alpha}=[a, \mu]^{T}$. The solution can be obtained by using the least square method $\hat{\alpha}=\left(\mathrm{B}^{\mathrm{T}} \mathrm{B}\right)^{-1} \mathrm{~B}^{\mathrm{T}} \mathrm{Y}_{\mathrm{n}}$, where: 


$$
Y_{n}=\left[\begin{array}{c}
X^{(0)}(2) \\
X^{(0)}(3) \\
\vdots \\
X^{(0)}(\mathrm{n})
\end{array}\right] \mathrm{B}=\left[\begin{array}{cc}
-Z^{(1)}(2) & 1 \\
-Z^{(1)}(3) & 1 \\
\vdots & \vdots \\
-Z^{(1)}(\mathrm{n}) & 1
\end{array}\right] Z^{(1)}(\mathrm{k})=\left(X^{(1)}(\mathrm{k})+X^{(1)}(\mathrm{k}-1)\right) / 2, \mathrm{k}=2,3, \ldots, \mathrm{n}
$$

After we get $\hat{\alpha}=[a, \mu]^{T}$, we can get the forecasting value of $\mathrm{X}^{(1)}$ :

$$
\hat{X}^{(1)}(\mathrm{k}+1)=\left[\hat{X}^{(0)}(1)-\frac{\mu}{\alpha}\right] e^{-\alpha k}+\frac{\mu}{\alpha}, k=1,2 \ldots
$$

Then we get the forecasting value of original data:

$$
\widehat{\mathrm{X}}^{(0)}(\mathrm{k}+1)=\widehat{\mathrm{X}}^{(1)}(\mathrm{k}+1)-\widehat{\mathrm{X}}^{(1)}(\mathrm{k}), \mathrm{k}=1,2 \ldots
$$

Metabolic GM $(1,1)$ Model. New data has more impact of the forecast value ${ }^{[3]}$. In original GM (1, 1) model, only the original data is used to forecast but not the new time series data, so the prediction accuracy will be greatly reduced with time. The basic principle of the metabolic GM $(1,1)$ model is that: we continue to receiving new data, while abandoning the old data. Suppose that the original sequence: $X^{(0)}{ }_{k=n}=\left\{X^{(0)}(1), X^{(0)}(2), \ldots, X^{(0)}(\mathrm{n})\right\}$. When we get the real value of $X^{(0)}(\mathrm{n}+1)$ at $\mathrm{n}+1$ time, we put $X^{(0)}(\mathrm{n}+1)$ into the sequence, and abandoning the most early data $X^{(0)}(1)$ in the original sequence. Then the new sequence: $X^{(0)}{ }_{k=n+1}=\left\{X^{(0)}(2), \ldots, X^{(0)}(\mathrm{n}), X^{(0)}(\mathrm{n}+1)\right\}$ will be used for subsequent prediction. And so on until the predicted is completion.

\section{New Optimal GM $(1,1)$ Model}

The metabolic GM $(1,1)$ model can make full use of the latest data to predict. But it accepts of new data with no condition. The accuracy of the prediction results is greatly affected by the abnormal data in the original sequence. So we put forward a new model based on the metabolic GM $(1,1)$ model, called optimal GM $(1,1)$ model. Strategy of the optimal GM $(1,1)$ model is shown as below:

1) Suppose the original data sequence is $X^{(0)}{ }_{k=n}=\left\{X^{(0)}(1), X^{(0)}(2), \ldots, X(n)\right\}$ at time $n$. We forecast the value of the time $\hat{X}^{(0)}(n+1)$ with the original GM $(1,1)$ model.

2) At the $n+1$ time, we get the observation value $X^{(0)}(n+1)$, put $X^{(0)}(n+1)$ into the sequence, and abandon the most early data $X^{(0)}(1)$ in the original sequence. After that, we get the new sequence: $X_{k=n+1}^{(0)}=\left\{X^{(0)}(2), \ldots, X^{(0)}(\mathrm{n}), X^{(0)}(\mathrm{n}+1)\right\}$. It is same to the metabolic GM $(1,1)$ model. The new sequence $X^{(0)}{ }_{k=n+1}=\left\{X^{(0)}(2), \ldots, X^{(0)}(\mathrm{n}), X^{(0)}(\mathrm{n}+1)\right\}$ is recorded as Sequence 1 .

3) Different from the metabolic GM $(1,1)$ model, we add an alternative sequence: $X^{(0)^{\prime}}{ }_{k=n+1}=$ $\left\{X^{(0)}(2), \ldots, X^{(0)}(n), \hat{X}^{(0)}(n+1)\right\}$ recorded as Sequence 2 , where $\hat{X}^{(0)}(n+1)$ is the forecasting value of step 1$)$.

4) Then we forecast the value of $n+2$ time by using Sequence 1 and Sequence 2 respectively. We get the forecasting value $\hat{X}^{(0)}(n+2)$ of Sequence 1 and $\hat{X}^{(0)^{\prime}}(n+2)$ of Sequence 2 .

5) After we get the observation value $X^{(0)}(\mathrm{n}+2)$ of $\mathrm{n}+2$ time, we compare it with the two forecasting value $\hat{X}^{(0)}(\mathrm{n}+2)$ and $\hat{X}^{(0)^{\prime}}(\mathrm{n}+2)$.If the error of $\hat{X}^{(0)}(\mathrm{n}+2)$ is smaller, Sequence 1 was selected to generate the follow-up Sequence 1 and Sequence 2. The new Sequence 1 is: $X^{(0)}{ }_{k=n+2}=\left\{X^{(0)}(3), \ldots, X^{(0)}(\mathrm{n}+1), X^{(0)}(\mathrm{n}+2)\right\}$ and the new Sequence 2 is $: X^{(0)}{ }_{k=n+2}^{\prime}=$ $\left\{X^{(0)}(3), \ldots, X^{(0)}(\mathrm{n}+1), \hat{X}^{(0)}(\mathrm{n}+2)\right\}$

Otherwise, Sequence 2 was selected to generate the follow-up Sequence 1 and Sequence 2 . Then the new Sequence 1 is: $X^{(0)}{ }_{k=n+2}=\left\{X^{(0)}(3), \ldots, \widehat{X}^{(0)^{\prime}}(\mathrm{n}+1), X^{(0)}(\mathrm{n}+2)\right\}$ and the new Sequence 2 is: $X^{(0)}{ }_{k=n+2}^{\prime}=\left\{X^{(0)}(3), \ldots, \widehat{X}^{(0)^{\prime}}(\mathrm{n}+1), \hat{X}^{(0)^{\prime}}(\mathrm{n}+2)\right\}$.

6) The new Sequence 1 and the new Sequence 2 was used to forecast the value of $n+3$ time. The forecasting value of the new Sequence 1 is $\hat{X}^{(0)}(n+3)$ and the forecasting value of the new Sequence 2 is $\hat{X}^{(0)^{\prime}}(n+3)$. When we get the observation value $X^{(0)}(n+3)$ of $n+3$ time, we selected the sequence to generate the follow-up sequence and generate the new Sequence 1 and the new Sequence 2, as Step 5).

7) And so on, until the forecasting is complete. 


\section{Application of the Optimal GM $(1,1)$ Model for Heating Load Forecasting}

Experimental Data Set. The experimental data set used in this paper is the measured data of the 2014-2015 annual heating data of Jinan municipal steam heating system. The data is the time series data of No.11-16 buildings of one zone from the time 2014-12-01 00:00:00.The time series data sampling interval is one hour. There are 2 abnormal values in the data. The data is shown below:

Table 1. Heating monitoring time series data

\begin{tabular}{|c|c|c|c|c|c|}
\hline Time & $\begin{array}{l}\text { the accumulated heat } \\
(\mathrm{Kw})\end{array}$ & Remarks & Time & $\begin{array}{l}\text { the accumulated } \\
\text { heat }(\mathrm{KW})\end{array}$ & Remarks \\
\hline 1 & 6631716.00 & & 13 & 6651212.90 & \multirow{12}{*}{$\begin{array}{l}\text { abnormal value, the real } \\
\text { value is } 6661062.70\end{array}$} \\
\hline 2 & 6633328.30 & & 14 & 6652870.50 & \\
\hline 3 & 6634944.50 & & 15 & 6654516.00 & \\
\hline 4 & 6636564.60 & & 16 & 6656149.70 & \\
\hline 5 & 6638204.00 & & 17 & 6657784.60 & \\
\hline 6 & 6639837.90 & & 18 & 6659431.20 & \\
\hline 7 & 6641472.00 & & 19 & 4621062.70 & \\
\hline 8 & 6643080.60 & & 20 & 6662689.00 & \\
\hline 9 & 6644717.30 & & 21 & 6664313.70 & \\
\hline 10 & 6646340.30 & & 22 & 6665936.60 & \\
\hline 11 & 6647955.00 & \multirow[b]{2}{*}{$\begin{array}{l}\text { abnormal value, the real } \\
\text { value is } 6649569.00\end{array}$} & 23 & 6667554.60 & \\
\hline 12 & 8640569.00 & & 24 & 6669183.80 & \\
\hline
\end{tabular}

\section{Experimental Results and Analysis}

Table 3. The Forecasting Results Using the Real Data

\begin{tabular}{ccrrrrr}
\hline \multirow{2}{*}{ Time } & \multirow{2}{*}{ Real Data } & \multicolumn{2}{c}{ Metabolic GM $(1,1)$ Model } & \multicolumn{2}{c}{ Optimal GM $(1,1)$ Model } \\
\cline { 3 - 6 } & & Forecasting Value & \multicolumn{1}{c}{$\delta$} & Forecasting Value & $\delta$ \\
\hline 9 & 6644717.30 & 6644719.09 & -1.79 & 6644719.09 & -1.79 \\
10 & 6646340.30 & 6646350.83 & -10.53 & 6646350.83 & -10.53 \\
11 & 6647955.00 & 6647975.66 & -20.66 & 6647975.66 & -20.66 \\
12 & 6649569.00 & 6649589.52 & -20.52 & 6649589.52 & -20.52 \\
13 & 6651212.90 & 6651201.07 & 11.83 & 6651201.07 & 11.83 \\
14 & 6652870.50 & 6652828.67 & 41.83 & 6652828.67 & 41.83 \\
15 & 6654516.00 & 6654478.82 & 37.18 & 6654478.82 & 37.18 \\
16 & 6656149.70 & 6656130.30 & 19.40 & 6656130.30 & 19.40 \\
17 & 6657784.60 & 6657783.12 & 1.48 & 6657783.12 & 1.48 \\
18 & 6659431.20 & 6659432.54 & -1.34 & 6659432.54 & -1.34 \\
19 & 6661062.70 & 6661079.98 & -17.28 & 6661079.98 & -17.28 \\
20 & 6662689.00 & 6662711.96 & -22.96 & 6662711.96 & -22.96 \\
21 & 6664313.70 & 6664335.82 & -22.12 & 6664335.82 & -22.12 \\
22 & 6665936.60 & 6665959.04 & -22.44 & 6665959.04 & -22.44 \\
23 & 6667554.60 & 6667580.10 & -25.50 & 6667580.10 & -25.50 \\
24 & 6669183.80 & 6669194.37 & -10.57 & 6669194.37 & -10.57 \\
\hline
\end{tabular}

First, we forecast with real value, without considering the abnormal data. As shown in Table 3, the forecasting result of optimal GM $(1,1)$ model are the same as Metabolic GM $(1,1)$ model. Table 4 shows that both models are Class I.

Table 4. Accuracy of Metabolic GM (1, 1) Model and Optimal GM (1, 1) Model

\begin{tabular}{lccl}
\hline Forecasting model & P & C & Accuracy class \\
\hline metabolic GM $(1,1)$ model & 1 & 0.0027651 & I \\
optimal GM $(1,1)$ model & 1 & 0.0027651 & I \\
\hline
\end{tabular}

Second, abnormal data in line12 and 19 are considered. As Fig.1 and Fig.2 show, after the abnormal acquisition value, the metabolic GM $(1,1)$ model is affected by the abnormal values until the sliding windows past the exception value. So the accuracy of the metabolic GM $(1,1)$ model is significantly lower. The optimal GM $(1,1)$ model only has one deviation after the abnormal data 
appears. The optimal GM $(1,1)$ model using the forecasting value replace the abnormal value to generate the follow-up sequence. The optimal GM $(1,1)$ model can quickly eliminate the impact of abnormal data item. So it has a good prediction accuracy.

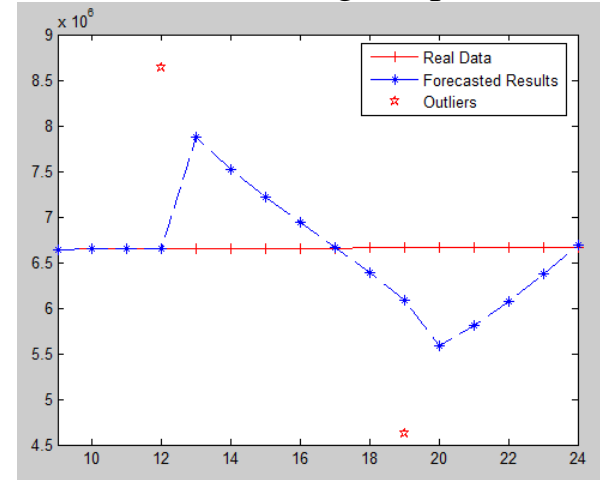

Fig.1 Result of metabolic GM $(1,1)$ model

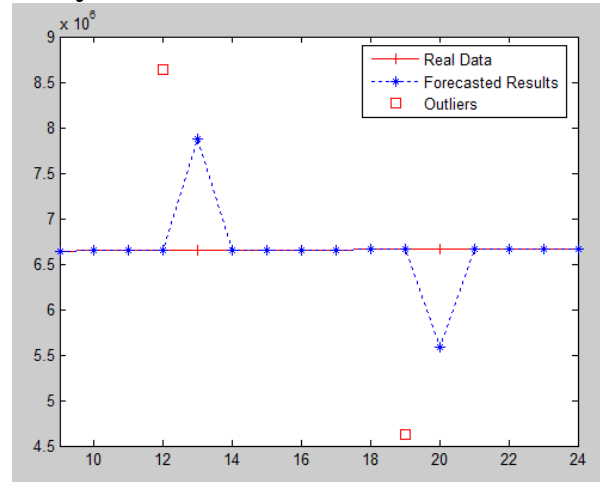

Fig.2 Result of optimal GM $(1,1)$ model

\section{Applicability Analysis of Optimal GM (1, 1) Model}

Experimental results show that if the original data contains some abnormal data, the optimal GM $(1,1)$ model can quickly eliminate the influence of abnormal data. So the prediction accuracy is much higher than that of the metabolic GM $(1,1)$ model. In this paper, the optimal GM $(1,1)$ model is used to predict 1 steps every time. In fact, the optimal GM $(1,1)$ model can be used to predict the multiple steps every time in order to apply to medium and long-term forecast.

\section{Conclusions}

In this paper, we present the optimal GM $(1,1)$ model based on the metabolic GM $(1,1)$ model. With the increase of the candidate sequences, the optimal GM $(1,1)$ model selected the prediction data or the real data are selected for the next step forecasting, which can avoid the prediction errors caused by the abnormal data and thus improve the prediction accuracy. The method is applied to forecasting heating load of municipal heating system.

\section{Acknowledgment}

The authors would like to acknowledge the support provided by the project of Shandong Province Higher Educational Science and Technology Program (J15LN53).

\section{References}

[1] Zhang Yongming. Nonlinear forecasting approach of heating load[D]. Harbin Institute of Technology, 2010 (in Chinese).

[2] Liu Sifeng, Zeng bo, Liu Jiefang, et al. Several basic models of GM(1,1) and their applicable bound[J]. Systems Engineering and Electronics, 2014,36(3):50-58 (in Chinese).

[3] Hsu C C, Chen C Y. Applications of improved grey prediction model for power demand forecasting[J]. Energy Conversion and Management, 2003, 44(14): 2241-2249.

[4] Chirwa E C,Mao Mingzhi. Application of grey model GM(1,1)to vehicle fatality risk estimation[J]. Technological Forecasting and Social Change,2006,73(5):588-605.

[5] Hao Yonghong,Zhao Jiaojuan, Li Huamin, et a1. Karst hydrological processes and grey system model[J]. Journal of the American water Resources Association,2012,48(4):656-666.

[6] Yang Jie, Weng Wenguo. Improved unbiased grey model for prediction of gas supplies[J]. Joural of Tsinghua University (Science and Technology),2014,54(2):145-148 (in Chinese). 